\title{
Basic Elements and Model Comparisons in Electrodynamics: Lightning Trajectory.
}

\author{
Odim Mendes Jr. \\ Osmar Pinto Jr \\ Iara R.C.A. Pinto \\ Mary Chryssafidis
}

Instituto Nacional de Pesquisas Espaciais (DGE/INPE) - 12201-970 São José dos Campos, SP, BR

odim@dge.inpe.br - Phone: (012) 345-6787 - FAX: (012) 345-6810

This paper presents the basic electrodynamics elements for studying the atmospheric behaviour and allowing the simulation of lightning trajectory. The principal electrodynamics models are presented and from the comparisons their major distinctive goals are briefly pointed out. The authors have identified that, though several models have been developed, it is necessary to obtain a realistic simulation model yet.

\section{INTRODUCTION}

Lightning consists basically of a high current ( kA), transient atmospheric electric discharge with path length around several kilometers. It is due to a great amount of electric charge $(\sim 10-100 \mathrm{C})$ accumulated in thunderclouds (cumulonimbus) and occurs when the electric field exceeds locally the air electric insulation $(>400 \mathrm{kV} / \mathrm{m})$. There are five types of lightning discharges: intracloud, cloud-to-ground, cloud-to-cloud, from cloud to air surrounding it and from cloud top towards stratosphere. To human environment cloud-to-ground lightning is the most important although observations have shown that its occurrence is very much less than the intracloud one.The main stages of cloud-to-ground lightning are essentially the preliminary breakdown, the stepped leader, the connecting leader and the return stroke, however subsequent stages may also occur (Volland, 1984; Uman, 1987).

In this paper we are interested in the development of stepped leader, which establishes the atmospheric ionized path to the complete lightning. Thus we consider basic elements of atmospheric electrodynamics, the models for simulation of lightning trajectory and their main features.

\section{BASIC ELEMENTS}

In general for obtaining a more complete lightning discharge model, we assume that the stepped leader progresses in the direction of the maximum potential gradiente at its tip. Due to involved problem, physical approximations are adopted so that the electric field can be found as the gradient of a scalar potential $(\mathbf{E}=-\nabla \varphi)$.

In such a case, we have the following equation for the scalar potential (Anderson and Freier, 1969; Pinto, 1987):

$\nabla^{2 \phi} / \mathrm{T}_{\mathrm{c}}-1 / \tau\left(\nabla^{2} \phi+2 \mathrm{~K} \partial \phi / \partial \mathrm{z}\right)=\nabla \cdot \mathrm{J}_{\mathrm{S}} / \varepsilon_{\mathrm{O}}$,

where $T_{c}$ is the characteristic time of the process; $\tau$ is the relaxation time of the air, given by
$\tau=\varepsilon_{\mathrm{O}} / \sigma ; \sigma$ is the atmospheric conductivity; $\mathrm{J}_{\mathrm{S}}$ is the current density of the charge source and $\varepsilon_{0}$ is the permittivity of free space. The conductivity is assumed to have an exponential profile given by $\sigma=\sigma_{\mathrm{O}} \exp (2 \mathrm{Kz})$, where $\mathrm{z}$ is the altitude, $(2 \mathrm{~K})^{-1}$ is the conductivity scale height and $\sigma_{\mathrm{O}}$ is the conductivity of the ground. In general, $\sigma_{\mathrm{O}}$ is between $10^{-15}$ and $10^{-13}$ $\mathrm{mho} / \mathrm{m}$. It is worth noting, however, that the solution of equation (1) does not depend on $\sigma_{\mathrm{o}}$. In fact, such a conductivity profile is a reasonable approach just to clear air. The magnitude and variation of the conductivity in the cloud environment of a thunderstorm are open to considerable questions. Some authors have considered that the conductivity inside the cloud is reduced by a factor of about 2 or 3 concerning the same altitude outside (Kasemir, 1965; Tzur and Roble, 1985). In some works, authors have considered for simplicity the conductivity inside the cloud equal to that outside, since otherwise equation (1) would not have analytical solution.

For lightning discharges $\mathrm{T}_{\mathrm{c}}$ is very small (less than one second), and only the first term in the right side of the equation (1) needs to be considered. The resulting equation has the coulomb solution. To this solution, we have added the solution of equation (1) just before the lightning discharge, as an initial condition, which corresponds to the charge separation process inside the cloud. This initial condition is obtained from equation (1) considering just the second term in the right side, since in this case $T_{c}$ is relatively long (tens of minutes). The resulting equation has the following solution

$\phi=1 / 4 \pi \varepsilon_{0} \sum \mathrm{q}_{\mathrm{i}} / \mathrm{R}_{\mathrm{i}} \exp \left(-\mathrm{K}\left[\mathrm{R}_{\mathrm{i}}+\Delta \mathrm{z}_{\mathrm{i}}\right]\right)$,

where

$\mathrm{R}_{\mathrm{i}}=\left[\mathrm{r}^{2}+\left(\mathrm{z}-\mathrm{z}_{\mathrm{i}}\right)^{2}\right]^{1 / 2}, \Delta \mathrm{z}_{\mathrm{i}}=\mathrm{z}-\mathrm{z}_{\mathrm{i}}$,

the index $\mathrm{i}$ accounts for the charge $\mathrm{q}_{\mathrm{i}}$ and its image, and $\mathrm{r}$ and $\mathrm{z}$ are the cylindrical coordinates of a point in the atmosphere. It is worth noting that during the lightning 
discharge, the potential given by equation (2) should remain constant, since the atmospheric response to the field changes $(\tau)$ is much slower than the duration of the discharge. This combined solution allows us to establish the electrodynamical analysis for the atmosphere and, in special case, to simulate the trajectory of lightning.

\section{MODEL COMPARISONS}

We analysed the different models for the atmospheric electrodynamics presenting their principal distinctive goals now.

The work developed by Anderson and Freier (1969) established the theoretical formalism (as earlier presented) necessary to study the lightning trajectory in the atmosphere. Considering the fast changes of lightning discharges compared with the atmospheric quasi-stationary situation, the authors obtained the potential distribution for a point vertical dipole with a constant conducting atmosphere and with an exponentially increasing conductivity and also the potential changes during the raising of a positive charge upward from the earth which eventually neutralizes the lower dipole charge. With this model the authors obtained a convective mechanism for thunderstorm regenerative charging.

Considering the vertical and tilted dipole configurations in the atmosphere, Takagi et al. (1986) computed the increase in the fraction of positive ground flashes occurring for various locations of lightning charges in a thundercloud related to dipole inclination. They used a stepped leader model in which a streamer, starting from an infinte cylindrical charge, progresses in the direction of maximum potential gradient at its tip. They had obtained a mechanism for high percentage of positive lightning at middle latitudes.

Considering the lightning at about one kilometer above from the point of striking on the ground, Dellera and Garbagnati (1982) provided a model for progression of the negative downward channels towards the earth and in inception and propagation of upward positive channels from earthed structures. They used a ring unipolar charges for the clouds, line segment charges for downward and upward leaders and point or line charges for the earthed electrodes form which it is supposed that the upward leaders can develop. Adopting a fractal technique Kawasaki et al. (1989) improved this model by adding a certain tortuosity and branches as representative characters of lightning channel. Those works allow evaluation of the exposure of structures located in flat territory and in different orographic conditions.

Takeuti et al. (1993) presented a recent different empirical approach to the problem of the calculation of the stepped path in the atmosphere, not considering the electric nature of the phenomena but a statistical method. A stepped leader to the ground under cloud base is modelled on the basis of photographs of ground discharges. This work analysed the shielding effect by high structures as towers.
Finally Mendes et al. (1996) have improved a model similar to Takagi's. They have considered a finite charged sphere in the thundercloud and the exponential increase of the atmospheric conductivity with altitude. This model has showed that the variable conductivity also increases the occurrence of positive lightning even for a vertical dipole. That result is in accordance to experimental results (Pinto et al., 1996) in the lowest latitudes. Thus this work establishes a more complete model and extends the mechanism which leads to a higher percentage of positive lightning. Presenting the occurrence of positive lightning, Figure 1 gives an example of the results obtained in this study compared to the Takagi's results.
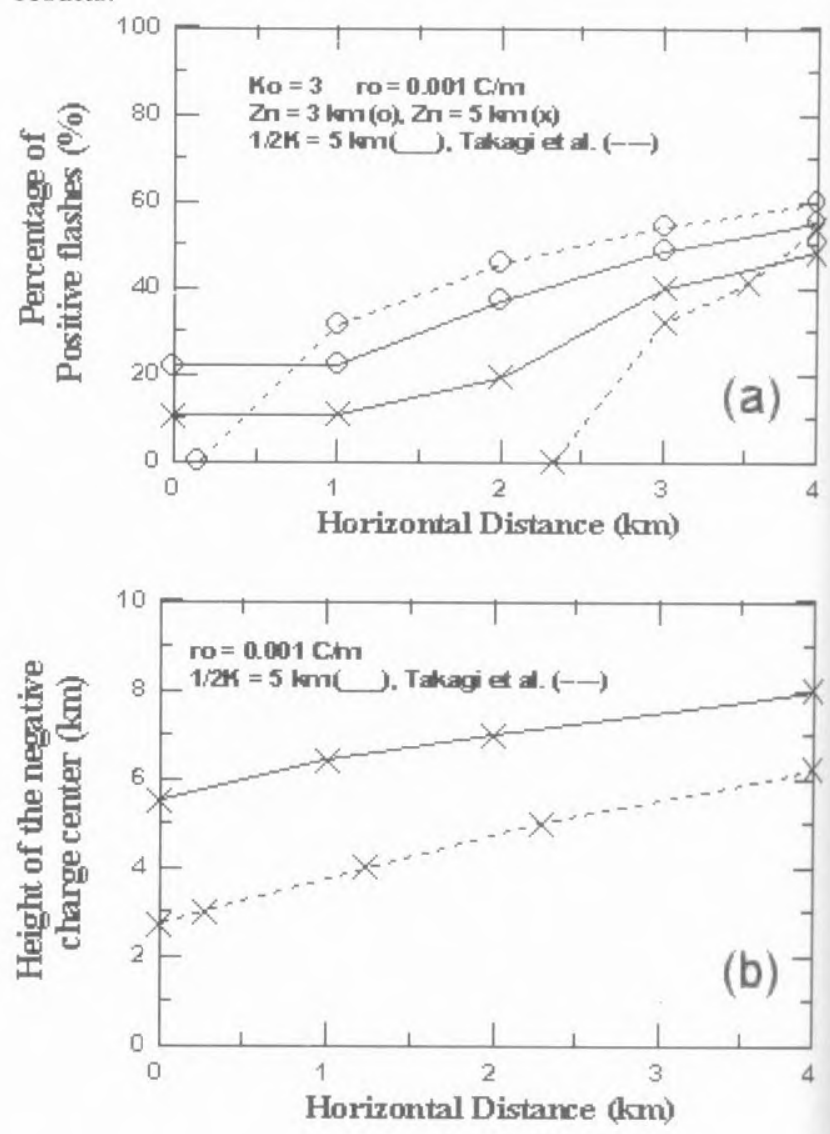

Fig. 1 - (a) The percentage of positive flashes versus the horizontal displacement of the positive charge with respect to the negative charge obtained by Mendes et al. (1996), considering a conductivity scale height of $5 \mathrm{~km}$, and by Takagi et al. (1986), for a constant conductivity. Values for two different altitudes of the negative charge ( 3 and $5 \mathrm{~km}$ ) are presented. The positive charge is located $3 \mathrm{~km}$ above the negative charge. Both models assume the same values of ro and Ko (see the papers for details). (b) The negative charge location for the occurrence of positive ground flashes obtained by Mendes et al. (1996) (conductivity scale height of $5 \mathrm{~km}$ ) and by Takagi et al. (1986). Positive flashes occur in the region below the curves.

\section{CONCLUSIONS}

Last 30 years several models improved our undestanding of atmospheric electrodynamics 
behaviour. Since they are limited to specific cases it is necessary to obtain a more complete lightning simulation model yet. All those models with their theoretical and experimental bases and with distinctive features point out to us the convenience and the possibility of obtaining a realistic electrodynamical model for lightning trajectory.

\section{REFERENCES}

Anderson, F. J., and G. D. Freier, Interactions of the thunderstorm with a conducting atmosphere. J. Geophys. Res., 74: 5390-5396, 1969.

Dellera, L.; Pigini, A.; Garbagnati, E. Lightning Simulation by Means of a Leader Progression model. International Conference on Lightning and Static Electricity. Proceedings. 22-35. St. Catherine's College, Oxford, 1982.

Kasemir, H. W. , The thundercloud, in Problems of atmospheric and space electricity, ed. S. C. Coroniti, Elsevier, Amsterdam, 1965.

Kawasaki, Z.I.; Matsuura, K.; Hasegawa, T.; Takeuti, T.; Nakano, M. Fractal Model for the Leader of Lightning. Res. Lett. Atmos. Electr., 9: 63-71, 1989.
Mendes Jr., O.; Pinto Jr., O.; Pinto, I.R.C.A; Chryssafidis, M. Lightning Simulation: The Stepped Leader Paths in the Earth's Atmosphere. VI Brazilian Plasma Astrophysics Workshop. Proceedings 1, 150153, São paulo, SP, Brazil, 1996.

Pinto, I.R.C.A. Estudo sobre campos elétricos e condutividade associados a nuvens eletrificadas na região da América do Sul. $\mathrm{PhD}$ Thesis, Instituto Nacional de Pesquisas Espaciais, São José dos Campos, Brazil, 1987. (INPE-4487-TDL/325).

Takagi, N., T. Takeuti, and T. Nakai, On the occurrence of positive ground flashes. J. Geophys. Res., 91:9905-9909, 1986.

Takeuti, T.; Hashimoto, T.; Takagi, N. A Computer Simulation on Lightning Stepped Leader Striking to Towers. VIII International Symposium on High Voltage Engineering. Proceedings. 265-267. Yokohama, Japan, 1993.

Tzur, I. , and R. G. Roble, The interaction of a dipolar thunderstorm with its global electrical environment. J. Geophys. Res., 90:5989-5999, 1985.

Uman, M. A., The lightning discharge, Academic Press, Florida, pp. 377, 1987.

Volland, H. Atmospheric Electrodynamics, SpringVerlag, New York, pp.205, 1984 